\title{
Youla-Kucera Parameterized Adaptive Regulator for Assistant Force Training in Lower Extremity Rehabilitation Robot System
}

\author{
Feng $\mathrm{Li}^{1}$, Zhizheng $\mathrm{Wu}^{2}$ and Jinwu Qian ${ }^{2}$ \\ ${ }^{1}$ School of Optical Electrical and Computer Engineering, University of Shanghai \\ for Science and Technology, Shanghai China, 200093 \\ ${ }^{2}$ Department of Precision Mechanical Engineering, Shanghai University, \\ Shanghai China, 200072 \\ E-mail:lifenggold@163.com
}

\begin{abstract}
Rehabilitation training is the major therapy in patients with hemiplegia or stroke and other limb locomotion dysfunction due to neurological or orthopedic problems. Taskoriented repetitive movements can improve motor performance in patients with neurological lesions. In this paper, considering the patient-cooperative intention, an adaptive regulator for Lower extremity rehabilitation robot (LERR) is proposed to control the robot's gait online to track the patient unknown joint trajectory with a desired assistant training force upon the patient. The design of the regulator is formulated within a Youla-Kucera parameterized set of stabilizing controllers and an adaptive algorithm is developed to search online the optimal parameters in the controller so that the patient unknown joint gait trajectory can be tracked and the desired assistant training force can be produced. The performance of the designed controller is verified in the Adams-Matlab co-simulation environment and the results illustrate the capability of the designed adaptive regulator for LERR to offer an effective assistant training force on the patient with unknown joint trajectory.
\end{abstract}

Keywords: Lower Extremity Rehabilitation Robot, adaptive regulator, Youla-Kucera parameterized, assistant force training

\section{Introduction}

It has been proved that task-oriented repetitive movements can improve muscular strength and movement coordination in patients with impairments resulting from neurological or orthopedic problems due to brain plasticity [1-3]. Weight-supported treadmill training has been shown to improve gait and lower limb motor function in patients with hemiplegia or stroke and other limb movement disorders [4]. Manually assisted treadmill training has been used for more than ten years as a regular therapy for patients with lower limb motor dysfunction. Numerous clinical studies support the efficacy of the training. Multiple studies have shown that weight-supported treadmill training can result in an improvement of balance, walking speed, endurance and other important gait characteristics. However, manual assisted treadmill training has server major limitations such as labor-intensive, low training duration, low training efficiency and low repeatability. In contrast, with automated gait training such as the robotic gait orthosis, these shortcomings mentioned above can be overcome.

Normally, the robotic gait orthosis works in so-called patient-cooperative or nocooperative way with respect to the cooperation with patients. In the no-cooperative way, the robot could not adapt the movement of the joints to the patient's contribution and the patient's limbs will be moved along a fixed reference trajectory. In the patient-cooperative way, the robot controller will recognize the patient's movement intention and motor abilities in terms of muscular efforts, then adapt the robotic assistance to the patient's 
contribution. More specifically, the robot will assist the patient's movement only as much as necessary and inform the patient how best to coordinate voluntary muscle efforts for a particular motion task.

For the robotic gait orthosis LOKOMAT [5], the impedance control and the admittance control methods have been considered in the control system to cooperate the patient's intentions. The basic idea of the impedance control or admittance control strategy is to allow a variable deviation from a predefined leg trajectory rather than imposing a rigid gait pattern. The amount of deviation depends on the patient's effort. An adjustable moment is applied at each joint to keep the leg within a defined range along the predefined trajectory. However, the main disadvantage of the impedance or admittance control strategy is they are based on a fixed reference trajectory, with the difficulty to perform large individual adjustments. In order to overcome these disadvantages, an adaptive control strategy [6] which can automatically adapt either the predefined reference trajectory of the reference-based controllers or the impedance magnitude of an impedance controller to the individual motion of a patient is proposed to allow the patient to influence the gait pattern. However, it could take a long time to adapt the reference trajectory to the desired one. The Hybrid force-position control method [7-8] which is highly patient-driven has been used for the LERR system to enable the patient to accomplish free walking movements and accelerate the rehabilitation progress. However, it is very difficult to obtain the desired reference trajectory for the position controller to ensure smooth and steady switching from the force control to the position control and the gait phase detection is complex. In [9], the control system of the Robotic Gait Rehabilitation Trainer with a novel mechanical design is presented, which targets secondary gait deviations affecting patterns of movement of the pelvis in stroke survivors. Similarly, an expanded impedance control strategy is applied to generate a force field that affects the obliquity of the pelvis via a lower body exoskeleton while the patient ambulates on a treadmill. A review of the current state of control methods for LERR can be found in [10-12].

The aforementioned control methods for the LERR system are mainly applied to the early rehabilitation stage at which the patient's reference gait trajectory is predefined or slightly modified by the patient's active force based on the standard human gait trajectory. However, in the final rehabilitation training stage, the patient joint movement intention and the resulting joint trajectory could be fully unknown. In such case, the robot needs to recognize the patient's movement intention online, and offer a necessary assistant training force on the patient to help to accomplish the desired walking movements. In this paper, a Youla-Kucera parameterized adaptive regulator design approach is proposed for the LERR system so that the patient unknown gait trajectory can be effectively tracked and the desired assistant training force is smoothly applied, and as well as the other unknown force disturbance produced from the contact environment is properly attenuated for the patient.

A six-degrees-of-freedom robotic orthosis called LERR(Figure1) has been built in Intelligent Machine and System Lab at Shanghai University. The LERR system mainly comprises powered gait orthosis(PGO) which is attached to the patient's legs, a bodyweight support system, a treadmill and a control system. Each hip, knee and ankle joint has one DOF and can realize flexion or extension movement in the sagittal plane which may simulate subject walking. Each joint is equipped with a linear actuator to drive the orthosis, a one DOF force sensor behind the linear actuator to measure the driving force and a rotary angle sensor to measure the joint angle. The gait orthosis can move upward and downward in the vertical direction while the patient's center of mass is fixed on parallelogram linkage. The dynamic model of LERR has been built and verified in[1314]. In this paper, an adaptive regulation approach is developed to modify the robot's gait trajectory online to track the patient unknown joint gait trajectory with a desired assistant training force. The proposed adaptive regulation approach consists of two main steps. 
First, a Youla-Kucera parameterized set of stabilizing controllers is constructed. Then, the parameter in the expression of stabilizing controllers is tuned online so that the controller converges to the desired controller needed to achieve gait trajectory regulation. The effectiveness of the proposed adaptive regulator is verified in the Adams-Matlab cosimulation environment, which shows the unknown patient intention gait trajectory can be tracked and the desired assistant training force can be produced to offer the necessary training force for the rehabilitation.

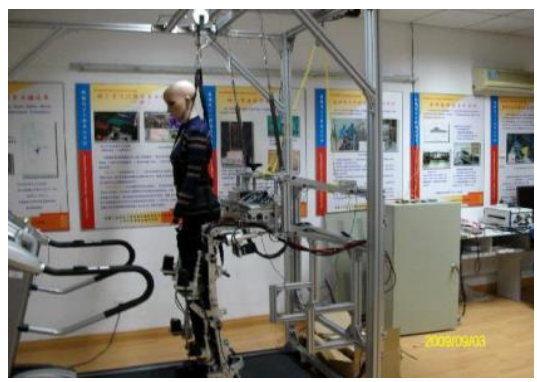

Figure 1. Lower Extremity Rehabilitation Robot with Six Degrees of Freedom

\section{Controller Design}

In this section, the robot system is first decoupled into three independent second-order integral sub-systems using invertibility decoupling method and an inner position control loop is designed based on decoupled robot dynamic model. Then, an adaptive regulator is further developed to deal with the patient cooperative intention, track the patient unknown joint gait trajectory and offer the desired assistant training force to the patient.

\subsection{The Position Control Design}

The LERR system is a complicated, strong coupling, nonlinear switched system. In order to simplify the controller design, the robot dynamic model is firstly decoupled using the standard invertibility decoupling method [15-16]. Since the gait cycle is divided into swing phase and stance phase, the dynamic model can be represented as:

$$
\sum_{r}\left\{\begin{array}{l}
M_{r}(\theta) \ddot{\theta}+C_{r}(\theta, \dot{\theta}) \dot{\theta}+G_{r}(\theta)=T \\
\left\{\begin{array}{l}
r=1, \text { in stance phase } \\
r=2, \text { in swing phase }
\end{array}\right.
\end{array}\right.
$$

Where, $\theta$ is three joint angle vectors, $M_{r}$ is a $3 \times 3$ inertia matrix, $C_{r}$ is a $3 \times 3$ centrifugal force and Coriolis force matrix, $G_{r}$ is a $3 \times 1$ gravity matrix, $T=T_{m}+T_{a c t}$ is a $3 \times 1$ joint torque required by the mechanical leg. $T_{m}$ is a drive torque offered by the motor, $T_{a c t}$ is an active torque produced by the patient. In stance phase, $r=1$, and in swing phase, $r=2$.

For the dynamic model in (1), we define

$$
\chi_{1}=\theta, \chi_{2}=\dot{\theta} \quad Y=\chi_{1}
$$

Then, the system is transformed into the following state space form as: 


$$
\left\{\begin{array}{l}
{\left[\begin{array}{l}
\dot{\chi}_{1} \\
\dot{\chi}_{2}
\end{array}\right]=\left[\begin{array}{c}
\chi_{2} \\
-M_{r}(\theta)^{-1} *\left(C_{r}(\theta, \dot{\theta}) \chi_{2}+G_{r}(\theta)\right)
\end{array}\right]+\left[\begin{array}{c}
0 \\
-M_{r}(\theta)^{-1} * T
\end{array}\right]} \\
\left\{\begin{array}{l}
r=1, \text { in stance phase } \\
r=2, \text { in swing phase }
\end{array}\right.
\end{array}\right.
$$

Take two times of derivative of $Y=\chi_{1}$ as

$$
\left\{\begin{array}{l}
\ddot{Y}=\dot{\chi}_{2}=-M_{r}(\theta)^{-1} *\left(C_{r}(\theta, \dot{\theta}) \chi_{2}+G_{r}(\theta)\right)+M_{r}(\theta)^{-1} * T \\
\left\{\begin{array}{l}
r=1, \text { in stance phase } \\
r=2, \text { in swing phase }
\end{array}\right.
\end{array}\right.
$$

Since $M_{r}(\theta)^{-1}$ is nonsingular, the whole system can be decoupled into 3 independent second-order integral sub-systems as

$$
v_{i}(t)=\ddot{y}_{i}(t), i=1,2,3
$$

Thus, the resulting system transfer function is $\frac{1}{s^{2}} I_{3 \times 3}$. The original control system is then converted into the following form:

$$
\sum_{c}\left\{\begin{array}{l}
u(t)=M_{r}(\theta) V(t)+C_{r}(\theta, \dot{\theta}) \dot{\theta}+G_{r}(\theta)-T_{a c t} \\
\left\{\begin{array}{l}
r=1, \text { in stance phase } \\
r=2, \text { in swing phase }
\end{array}\right.
\end{array}\right.
$$

where $u(t)=T_{m} \quad$ is a drive torque offered by the motor. The resulting decoupled closed-loop system is shown in Figure2.

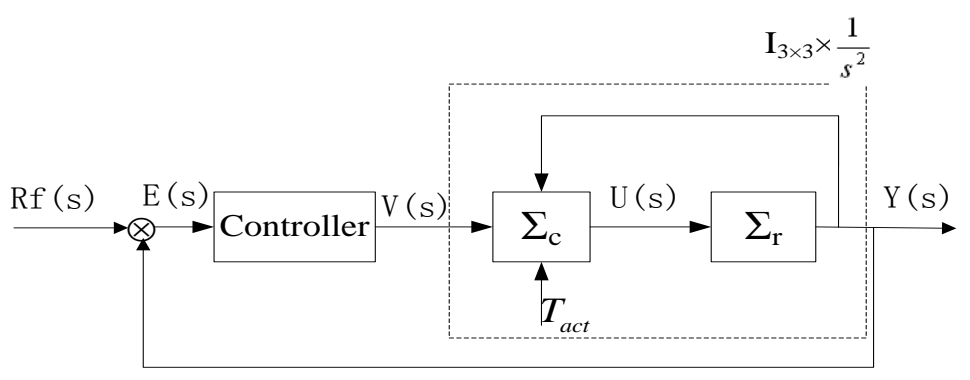

Figure 2. Block Diagram of the Inner Position Control Loop

Based on the invertibility controller (5), the system (1) is finally decoupled into three independent second-order integral sub-systems $\bar{G}(s)=\frac{1}{s^{2}} I_{3 \times 3}$. Then, for each channel, a decentralized PD or Lead-Lag position controller $K_{0}$ can be easily designed to make the output $Y$ effectively track the reference trajectory $R f$ in the inner control loop.

However, in the patient-cooperative way, the patient's intention movement trajectory is unknown, which implies the reference trajectory is unknown and possibly time-varying. In such case, the robot should be able to effectively track the patient unknown joint trajectory online, and at the same time could offer the desired assistant training force to the patient for accomplishing the rehabilitation training. Moreover, the other unknown disturbance factors upon the robot system, i.e. the disturbance forces resulting from the 
treadmill interaction or mechanical vibrations, should also be coped with properly. These force disturbances could be equalized as input disturbance added to the control input to the robot system. Therefore, the design object of the whole control system is formulated into a regulation problem which is to track the patient's unknown intention gait trajectory, offer the assistant training force, and attenuate unknown force disturbances resulting from the robot and the contact environment. This idea led to the development of the adaptive regulator for the LERR system in the following.

\subsection{Design of the Adaptive Regulator}

The aforementioned control problem in the LERR system can be formulated as a regulation problem as shown in Figure3, where $u$ is the control input, ${ }_{f}$ the equivalent force disturbance in the robot system, $d_{p^{\prime}}$ the patient unknown intention joint gait trajectory, ${ }^{d}$ the desired assistant training force offered by LERR to the patient, $e^{e}$ the assistant training force tracking error which is supposed to be zero. $F(s)$ is the transfer function of the force sensor, $\bar{G}(s)$ is the decoupled LERR control model. It is desired to construct an output feedback controller to regulate the performance variable $e$ against the unknown exogenous signal $d_{f} d_{p^{\prime}}$ and $d_{s}$ such that $\lim _{k \rightarrow \infty} e(k)=0$. The closed loop system block diagram in Figure3 can be redrawn as shown in Figure4. In the following, a Youla-Kucera $({ })$ parameterized adaptive regulator design approach is proposed. First, the base controller is designed to stabilize the closed loop system, then the base controller is parameterized with $Q$ parameters in a set of all stabilizing controllers. Finally, the adaptive algorithm is developed to online tune the $Q$ parameters to the desired one that can achieve regulation against the unknown exogenous signals and provide the desired assistant training force upon the patient.

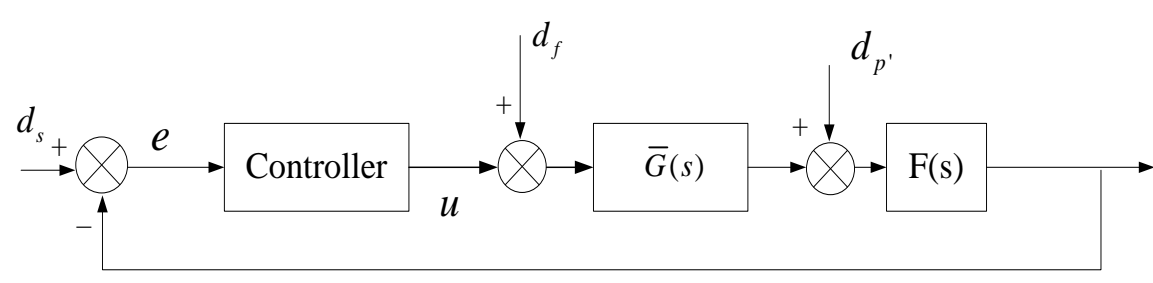

Figure 3. Control Block Diagram of the LERR System

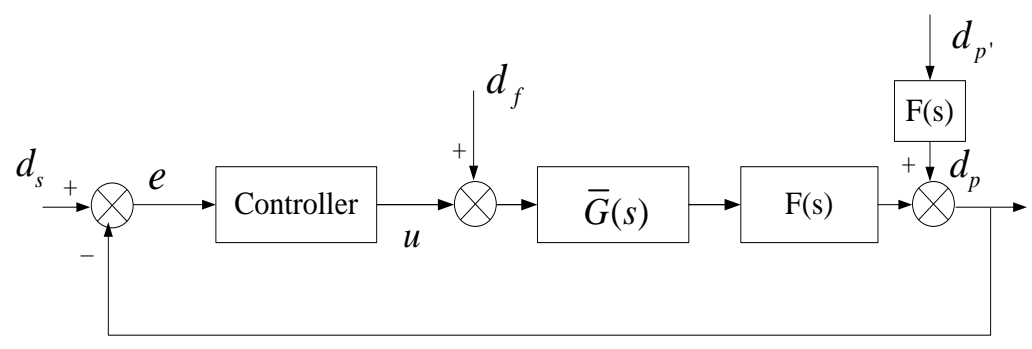

Figure 4. The Equivalent Control Block Diagram of the LERR System

\subsubsection{Youla-Kucera Parameterization of Stabilizing Controllers}


The main idea behind the adaptive regulator design proposed in this paper is to search online for the desired regulator within the set of parameterized stabilizing controllers for the LERR system to achieve regulation against the exogenous inputs $d_{f}, d_{p}$ and $d_{s}$.

Based on Section (2.1), the robot system has been decoupled into three independent second-order integral systems as $\bar{G}(s)=1 / s^{2} I$, where $I$ is $3 \times 3$ identity matrix. Denote $P(s)=\bar{G}(s) F(s)$, and the discrete-time representation of $P(s)$ as $P\left(z^{-1}\right)=\frac{A_{p}\left(z^{-1}\right)}{B_{p}\left(z^{-1}\right)}$, where $A_{p}\left(z^{-1}\right)$ and $B_{p}\left(z^{-1}\right)$ are polynomials in the complex variable $z^{-1}$. Assume there is a stabilizing controller $K_{0}$ for the plant $P\left(z^{-1}\right)$, which is given by $K_{0}\left(z^{-1}\right)=\frac{A_{k}\left(z^{-1}\right)}{B_{k}\left(z^{-1}\right)}$. Denote $M\left(z^{-1}\right)=\frac{1}{B_{p}\left(z^{-1}\right) B_{k}\left(z^{-1}\right)-A_{p}\left(z^{-1}\right) A_{k}\left(z^{-1}\right)}$, and $M \in R H_{\infty}$ is a stable transfer function. Using the base stabilizing controller $K_{0}$, the set of all stabilizing controllers can then be constructed based on the Youla-Kucera parameterization approach [17]. In fact, for any $Q \in R H_{\infty}$, the controller $K$ given by

$$
K=\frac{A_{k}\left(z^{-1}\right)+M\left(z^{-1}\right) B_{p}\left(z^{-1}\right) Q\left(z^{-1}\right)}{B_{k}\left(z^{-1}\right)+M\left(z^{-1}\right) A_{p}\left(z^{-1}\right) Q\left(z^{-1}\right)}
$$

is a stabilizing controller for the plant $P\left(z^{-1}\right)$. Moreover, every rational stabilizing controller $K$ has the form for some $Q \in R H_{\infty}$. Let $G\left(z^{-1}\right)$ be the transfer matrix of the augmented plant with inputs $\left[\begin{array}{l}d \\ u\end{array}\right]$ and outputs $\left[\begin{array}{l}e \\ y\end{array}\right]$, where $d=\left[\begin{array}{l}d_{f} \\ d_{p} \\ d_{s}\end{array}\right]$ is the disturbance vector, $u$ is the control input, $e$ is the performance variable to be regulated and ${ }^{y}$ is the measurement signal to be fed to the controller. Then given a stabilizing controller $K$ as in (6), the regulation problem against the disturbance $d$ can be formulated with the $J$ and $Q$ blocks as shown in Figure5. The $J$ block is given by

$$
J=\left[\begin{array}{cc}
K_{0}\left(z^{-1}\right) & \frac{1}{B_{k}\left(z^{-1}\right)} \\
\frac{1}{B_{k}\left(z^{-1}\right)} & -\frac{M\left(z^{-1}\right) A_{p}\left(z^{-1}\right)}{B_{k}\left(z^{-1}\right)}
\end{array}\right]
$$

For the LERR system, define $y=e$, which is the force tracking error signal and is supposed to be zero. Then the augmented plant $G$ can be represented as $G=\left[\begin{array}{ll}G_{11} & G_{12} \\ G_{21} & G_{22}\end{array}\right]$, where $G_{11}=G_{21}=\left[\begin{array}{lll}P & {[1} & 1\end{array}\right]$ and $G_{12}=G_{22}=P$. The two blocks 
$G$ and $J$ can be combined into a single block $T$ given by $T=\left[\begin{array}{ll}T_{11} & T_{12} \\ T_{21} & T_{22}\end{array}\right]$, where $T_{11}=G_{11}+G_{12} M A_{k} B_{p} G_{21}, T_{12}=G_{12} B_{p}, T_{21}=B_{p} G_{21}$ and $T_{22}=0$. In this paper, a weighted Ritz-type $Q$ parameter is considered as:

$$
\begin{gathered}
Q\left(z^{-1}\right)=\sum_{i=1}^{n_{q}} \theta_{i} z^{1-i} F\left(z^{-1}\right), \quad i=1, \cdots n_{q} \\
F\left(z^{-1}\right)=\frac{b_{1} z^{-1}+\cdots+b_{s} z^{-s}}{1+a_{1} z^{-1}+\cdots+a_{s} z^{-s}} \text { is used to adjust the }
\end{gathered}
$$

is used to adjust the dynamic properties of $Q\left(z^{-1}\right)$ and thereby optimally shape the sensitivity functions of the closed loop system.

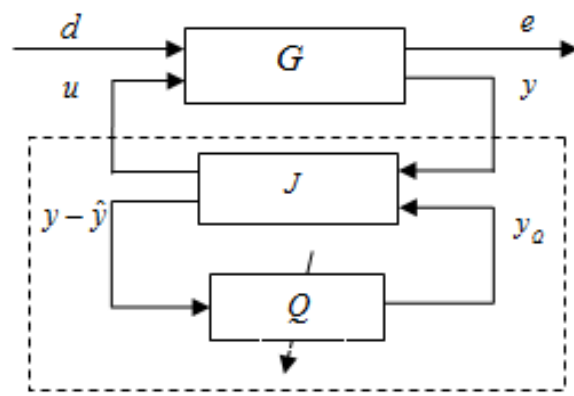

\section{Figure 5. Closed Loop System with the $Q$-Parameterized Controller}

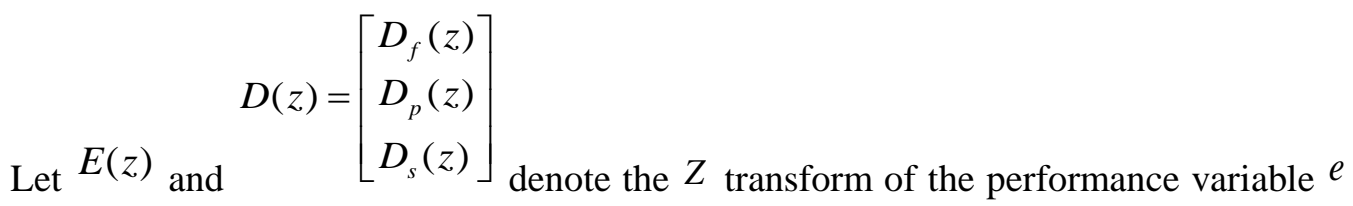
and the external input $d$, respectively. The closed loop system performance variable is then given by

$$
E\left(z^{-1}\right)=F_{T, Q}\left(z^{-1}\right) D\left(z^{-1}\right)=\left[T_{11}\left(z^{-1}\right)+T_{12}\left(z^{-1}\right) Q\left(z^{-1}\right) T_{21}\left(z^{-1}\right)\right] D\left(z^{-1}\right)
$$

Consider the weighted Ritz-type parameterization (7) and define the parameter vector $L=\left[\theta_{1}, \cdots, \theta_{n_{q}}\right]^{T}$, the linear interpolation condition can then be derived for achieving regulation against the disturbance $d$ in the closed loop system [17].

\subsubsection{Adaptive Algorithm}

Since the patient's gait trajectory $d_{p}$ and force disturbance $d_{f}$ are unknown and possibly time varying, it is desired to introduce adaptation in the controller design process. The aim of the adaptation is to tune the parameter vector $L$ so that it converges to the desired parameter vector $L_{0}$ needed to achieve regulation. Let $q^{-l}$ denote the $l$ time step delay operator, and $Q_{k}=\bar{Q}_{k} F\left(q^{-1}\right)$, where $\bar{Q}_{k}=\sum_{i=1}^{n_{q}} \theta_{i}(k-1) q^{1-i}$. The performance variable $e(k)$ is then given by 


$$
\begin{aligned}
e(k) & =\left[T_{11}\left(q^{-1}\right)+T_{12}\left(q^{-1}\right) \bar{Q}_{k} F\left(q^{-1}\right) T_{21}\left(q^{-1}\right)\right] d(k) \\
& =T_{11}\left(q^{-1}\right) d(k)+T_{12}\left(q^{-1}\right) \bar{Q}_{k} F\left(q^{-1}\right) r(k)
\end{aligned}
$$

where $r(k)=T_{21}\left(q^{-1}\right) d(k)=y(k)-\hat{y}(k) . r(k)$ is one of the outputs of the block $J$ and thus can be obtained at each step $k$. Let $L_{0}$ be a parameter vector satisfying the interpolation condition [17] and $\bar{Q}_{0}$ be the Youla parameter that results from using $L_{0}$. The corresponding performance error resulting from $\bar{Q}_{0}$ can then be written as

$$
e_{0}(k)=T_{11}\left(q^{-1}\right) d(k)+\bar{Q}_{0} T_{12}\left(q^{-1}\right) F\left(q^{-1}\right) r(k)
$$

and $\lim _{k \rightarrow \infty} e_{0}(k)=0$. Define the signals:

and the error

$$
\bar{e}(k)=\left[T_{12}\left(q^{-1}\right) \bar{Q}_{k}-\bar{Q}_{k} T_{12}\left(q^{-1}\right)\right] F\left(q^{-1}\right) r(k)
$$

$$
\begin{aligned}
& \tilde{e}^{0}(k)=e(k)-\bar{e}(k) \\
& =T_{11}\left(q^{-1}\right) d(k)+\bar{Q}_{0} T_{12}\left(q^{-1}\right) F\left(q^{-1}\right) r(k)+\left(\bar{Q}_{k}-\bar{Q}_{0}\right) T_{12}\left(q^{-1}\right) F\left(q^{-1}\right) r(k)
\end{aligned}
$$

Define $n(k)=T_{12}\left(q^{-1}\right) F\left(q^{-1}\right) r(k)$ and the regression vector

$$
\phi(k)=\left[-n(k), \cdots,-n\left(k-n_{q}+1\right)\right]^{T}
$$

Define the estimated parameter vector $\hat{L}(k)=\left[\hat{\theta}_{1}(k), \cdots, \hat{\theta}_{n_{q}}(k)\right]^{T}$ and $\tilde{L}(k)=L_{0}-\hat{L}(k)$, then the error $\tilde{e}^{0}(k+1)$ can be written as:

$$
\begin{gathered}
\tilde{e}^{0}(k+1)=\phi^{T}(k+1)\left(L_{0}-\hat{L}(k)\right)+e_{0}(k+1) \\
=\phi^{T}(k+1) \tilde{L}(k)+e_{0}(k+1)
\end{gathered}
$$

The corresponding posteriori error is

$$
\begin{gathered}
\tilde{e}(k+1)=\phi^{T}(k+1)\left(L_{0}-\hat{L}(k+1)\right)+e_{0}(k+1) \\
=\phi^{T}(k+1) \tilde{L}(k+1)+e_{0}(k+1)
\end{gathered}
$$

The estimation of the unknown parameter vector $\hat{L}(\cdot)$ can then be performed using a gradient adaptation algorithm or recursive adaptive algorithms. For example, the recursive least squares algorithm with a time varying forgetting factor can be given as follows:

$$
\begin{aligned}
\hat{L}(k+1) & =\hat{L}(k)+P(k) \phi(k+1) \frac{\tilde{e}^{0}(k+1)}{1+\phi(k+1) P(k) \phi^{T}(k+1)} \\
P(k+1) & =\frac{1}{\lambda(k+1)}\left[P(k)-\frac{P(k) \phi(k+1) \phi^{T}(k+1) P(k)}{1+\phi^{T}(k+1) P(k) \phi(k+1)}\right]
\end{aligned}
$$

with $\hat{L}(0)=0, P(0)=P_{0}>0$, and where $\lambda(k)$ is a time varying forgetting factor satisfying $0<\lambda_{\min } \leq \lambda(k) \leq \lambda_{\max }<1$. Since $T_{12}\left(q^{-1}\right), F\left(q^{-1}\right)$ and $T_{21}\left(q^{-1}\right)$ are all stable transfer functions, then if the disturbance vector $d$ is bounded, based on (11) it is easy to obtain that the regressive vector $\phi(k)$ is bounded. Thereby, based on Strict Positive Real (SPR) condition [18], if it is assumed that there exists a parameter vector $L_{0}$ satisfying the regulation condition [17] corresponding to the disturbance input properties, then the 
algorithm given by (14) yields $\lim _{k \rightarrow \infty} \hat{L}(k)=L_{0}$ and regulation against the disturbance $d$ in the adaptive closed loop system is achieved.

\section{Simulation Result}

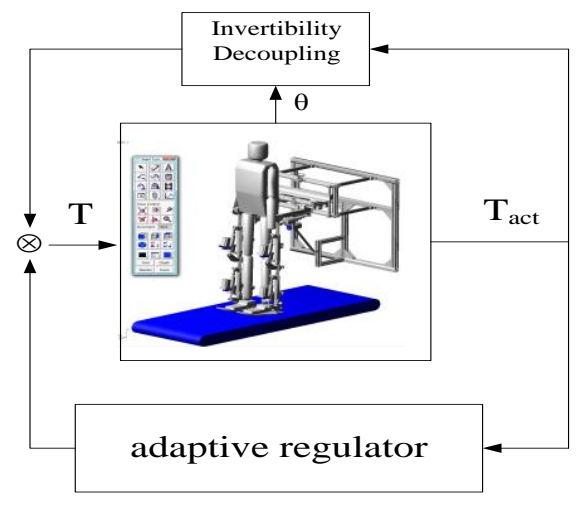

\section{Figure 6. Simulation in Matlab/Simulink and Adams}

In this section, the dynamic model of LERR is developed in Matlab/Simulink and Adams co-simulation environment (Figure6). The proposed adaptive regulator is evaluated to verify the capability of tracking the patient unknown joint gait trajectory, offering the desired assistant training force, and attenuating unknown force disturbances resulting from the external environment. The force sensor transfer function $F(s)$ is represented as $F(s)=\frac{1000}{0.01 s+1}$. The discrete-time augmented plant model $P$ is represented as

$$
P(z)=\frac{8.3 \times 10^{-3} z^{3}+2.5 \times 10^{-2} z^{2}+2.5 \times 10^{-2} z+8.3 \times 10^{-3}}{z^{3}-2.33 z^{2}+1.67 z-0.33}
$$

The stabilized base controller $K_{0}$ is designed as $K_{0}\left(z^{-1}\right)=\frac{250-230 z^{-1}}{1+z^{-1}}$. The $Q$ parameter is selected to be of the form

$$
Q\left(z^{-1}\right)=\sum_{i=1}^{6} \theta_{i} z^{1-i} F\left(z^{-1}\right)
$$
, where $F\left(z^{-1}\right)$ is a low pass filter with the cut off frequencies $20 \mathrm{~Hz}$. The force disturbance signal is simulated by a time-varying sinusoidal signal with the amplitude $5 \mathrm{~N}$ and with a constant offset $5 \mathrm{~N}$. Since the patient joint trajectory and the force disturbance are assumed to be unknown, the proposed adaptive algorithm is used to tune $L$ in the $Q$ parameter online to achieve regulation. The sampling period for the adaptive algorithm is $0.01 \mathrm{~s}$. The forgetting factor in the adaptation algorithm is set as a constant $\lambda=0.96$. The initial conditions for the adaptation algorithm are $\hat{L}(0)=\left[\begin{array}{llllll}0 & 0 & 0 & 0 & 0 & 0\end{array}\right]^{T}$ and $P(0)=10^{3} I_{6 \times 6}$ where $I_{6 \times 6}$ is $6 \times 6$ identity matrix. In the adaptation algorithm, the UD factorization algorithm is used in order to improve the numerical properties of the algorithm.

In following, only the results for the knee joint are illustrated. Firstly, it is assumed that the desired knee joint assistant training force offered by LERR is constant as shown in Figure7. The simulation results of the knee joint with the constant assistant training force using the adaptive regulator are shown as Figure8, Figure9 and Figure10. Figure8 shows the output trajectory of the knee joint, where the dashed line is the patient unknown 
movement trajectory and the solid line is the output trajectory of the robot with the adaptive regulator. Figure9 shows the force tracking error of the knee joint. Figure10 shows the parameter $L$ of the $Q$ parameterized adaptive regulator.

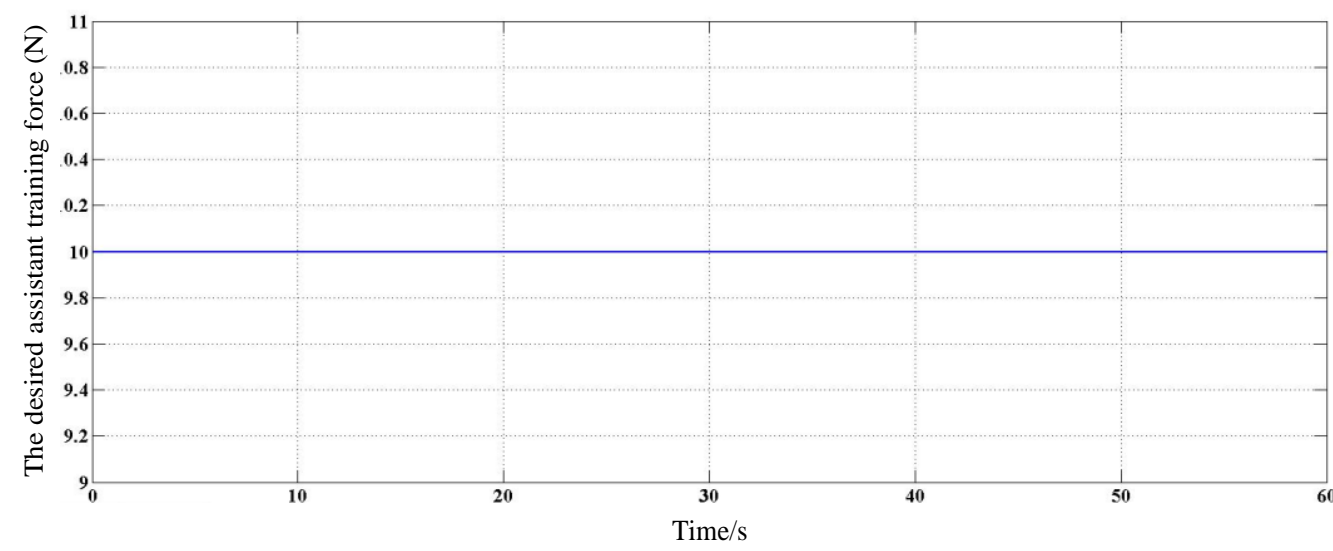

Figure 7. The Desired Constant Assistant Training Force Offered by LERR

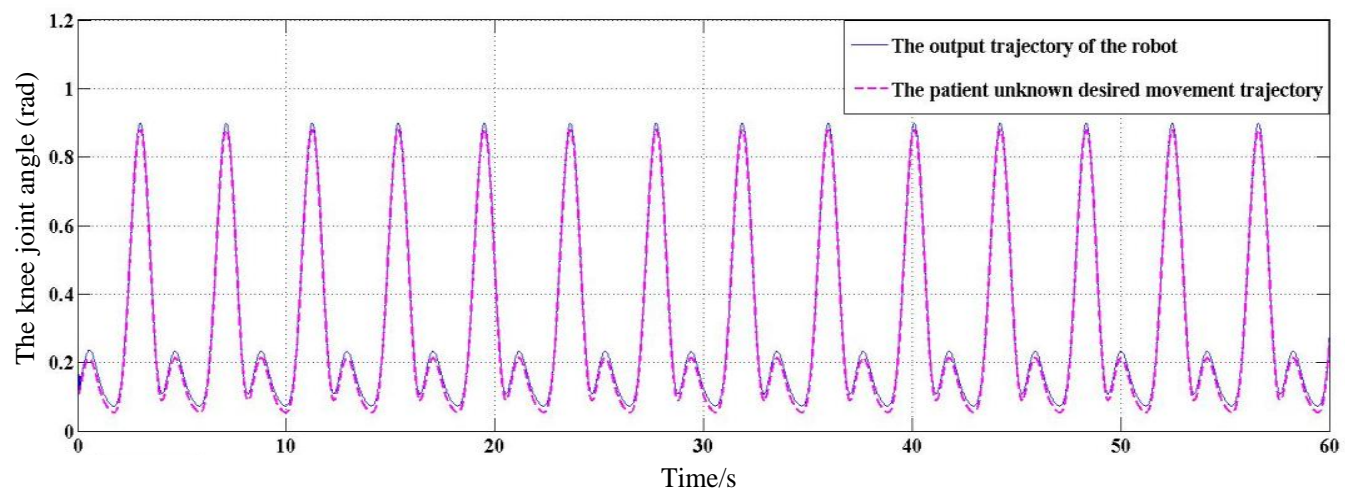

Figure 8. The Output Trajectory of Knee Joint with Constant Assistant Training Force Using $Q$ Parameterized Adaptive Regulator

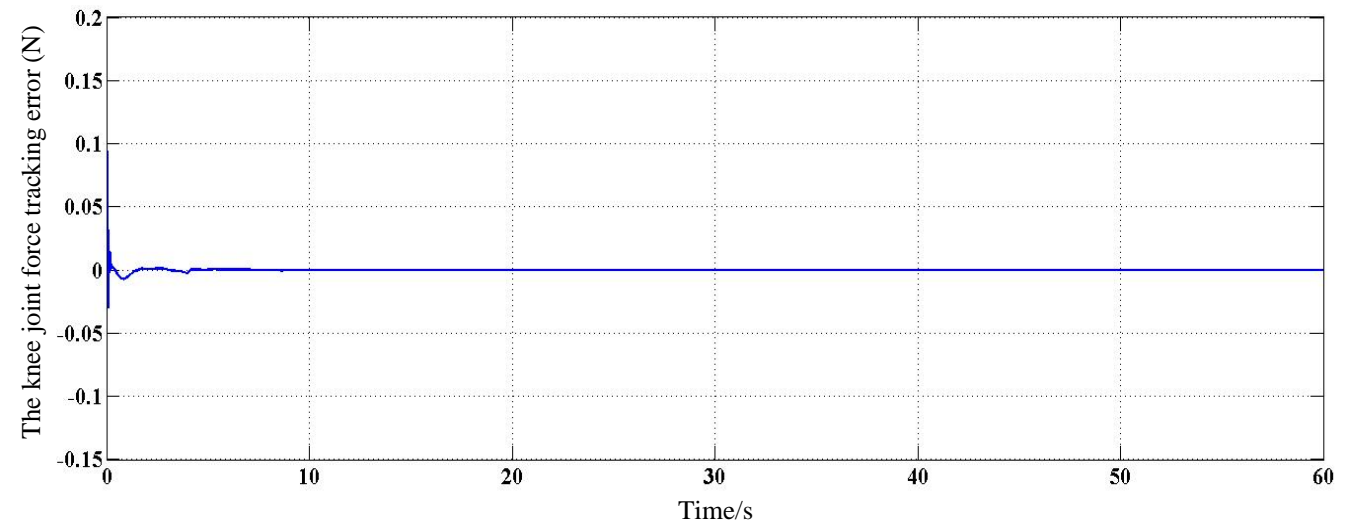

Figure 9. The Force Tracking Error of Knee Joint with Constant Assistant Training Force Using $Q$ Parameterized Adaptive Regulator 


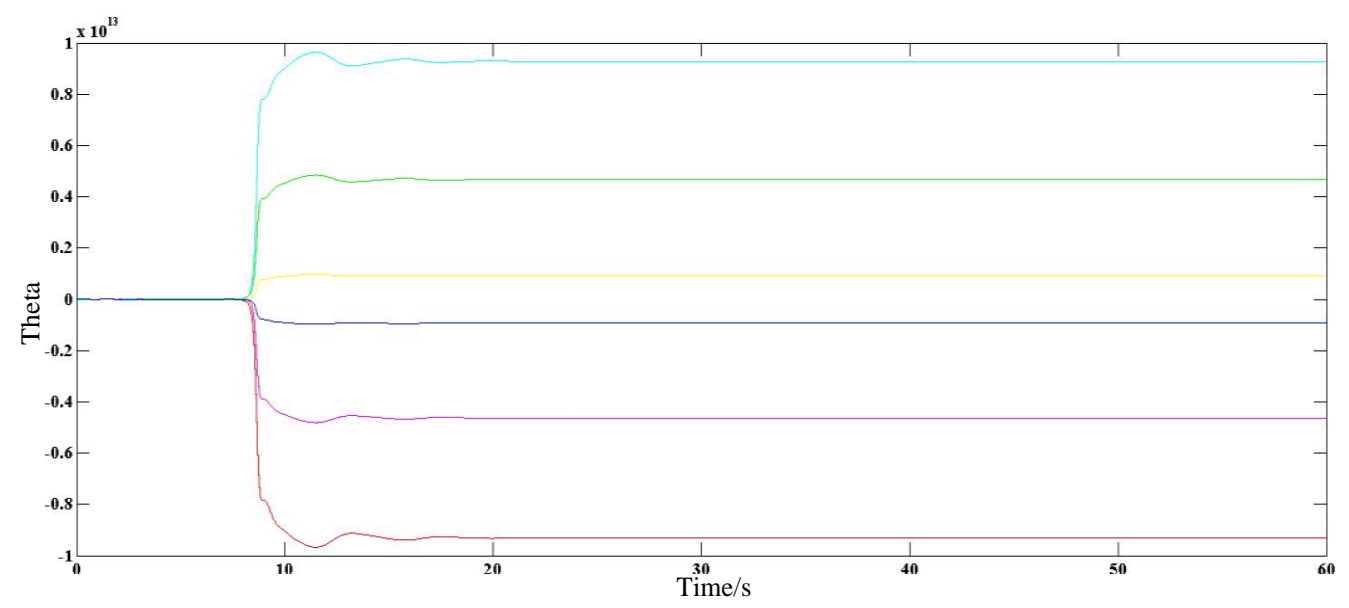

Figure 10. The Parameter $L$ of the $Q$ Parameterized Adaptive Regulator

Secondly, it is assumed that the desired assistant training force offered by LERR is time-varying as shown in Figure 11 and the unknown patient joint movement is also timevarying. The simulation results of the knee joint with the time varying patient joint trajectory and assistant training force using the adaptive regulator are shown as in Figure12, Figure13 and Figure14. Figure12 shows the output trajectory of the knee joint, where the dashed line is the patient unknown desired movement trajectory and the solid line is the output trajectory of the robot with the adaptive regulator. Figure 13 shows the force tracking error of the knee joint. Figure14 shows the parameter $L$ of the $Q$ parameterized adaptive regulator.

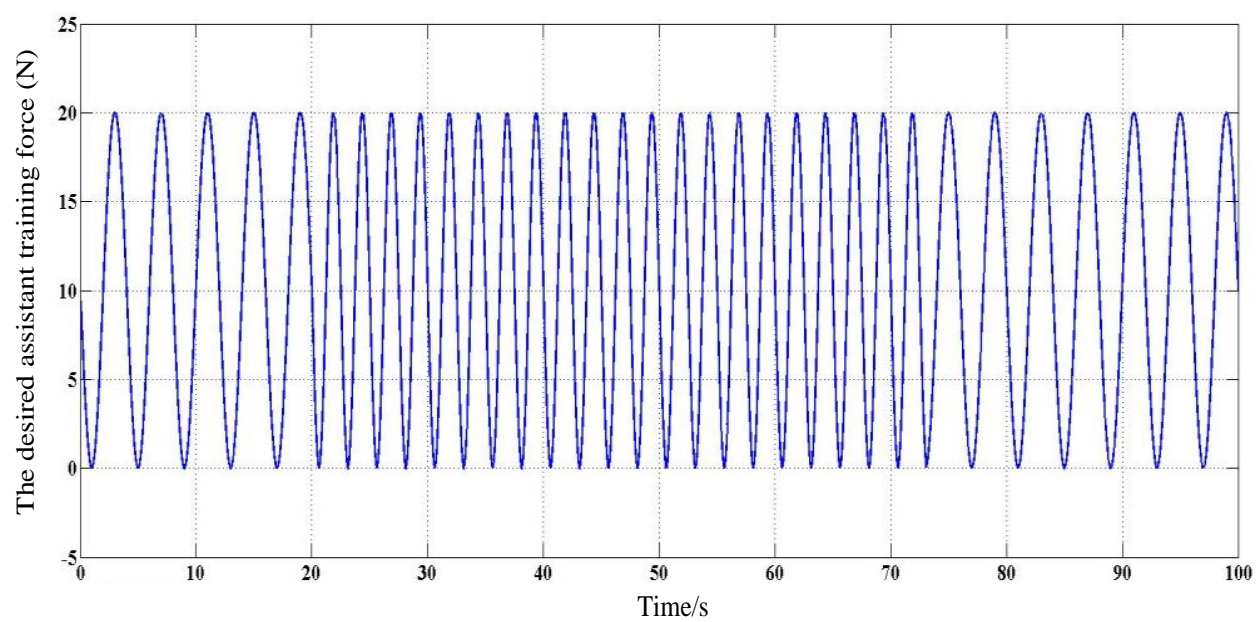

Figure 11. The Desired Time-Varying Assistant Training Force Offered by LERR 


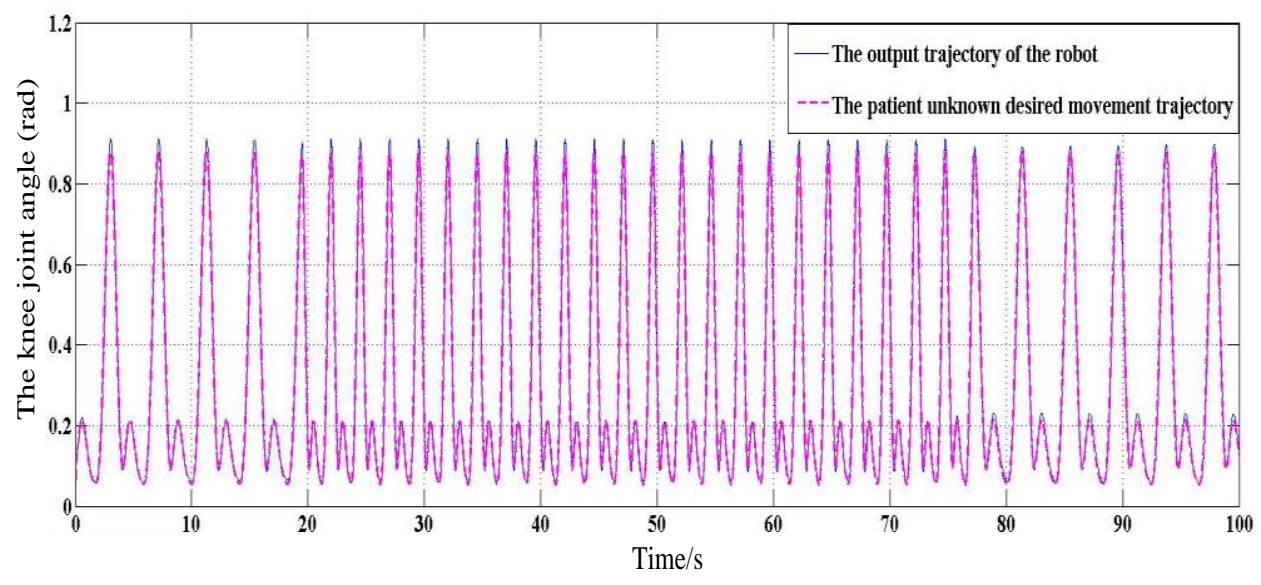

Figure 12. The Output Trajectory of Knee Joint with Time-Varying Patient Joint Trajectory and Assistant Training Force using $Q$ Parameterized Adaptive Regulator 


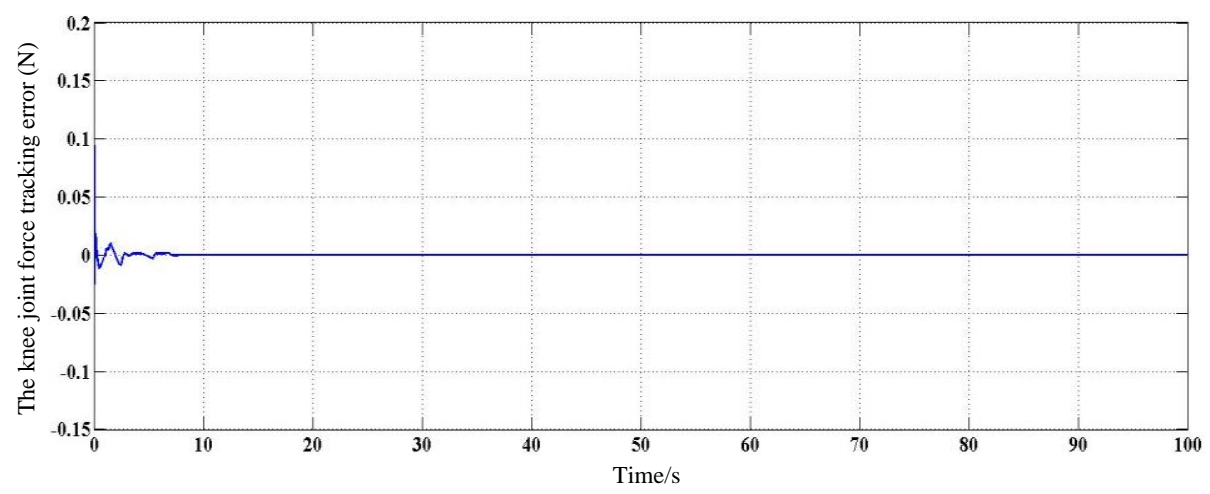

Figure 13. The Force Tracking Error of Knee Joint with Time-Varying Patient Joint Trajectory and Assistant Training Force using $Q$ Parameterized Adaptive Regulator

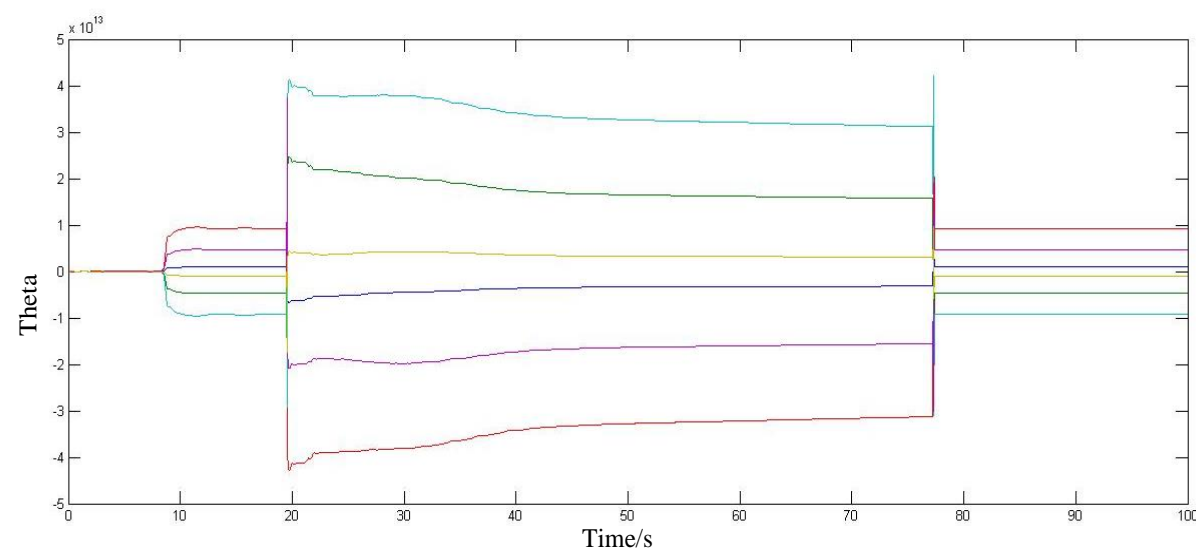

\section{Figure 14. The Parameter $L$ of the $Q$ Parameterized Adaptive Regulator}

It can be seen from the above simulation results that the output trajectory of the robot with the designed controller can effectively track the unknown patient movement trajectory, and the force tracking error can fast converge to zero so that the desired assistant training force is provided and the other unknown force disturbance is attenuated. Therefore, the proposed Youla-Kucera parameterized adaptive controller can be used to enhance the control performance of the LERR system in a smooth patient-cooperative way for handling the unknown properties in the rehabilitation training process.

\section{Conclusion}

Tracking patient's unknown gait trajectory and the generation of desired assistant training force are critical to the patient's cooperative rehabilitation training. In this paper, a Youla-Kucera parameterized ( $Q$ ) adaptive regulation approach is proposed to track the patient unknown gait trajectory and offer the patient the desired assistant training force despite the unknown exogenous disturbances in the system. The regulator design approach based on the Youla-Kucera parameterization of all stabilizing controllers is first presented. Then considering the patient's unknown movement intention, an adaptive approach is further proposed to tune the $Q$ parameter online in order to track the patient's unknown gait trajectory, attenuate the unknown force disturbance and offer the desired assistant training force. The performance of the proposed controller is evaluated in the Adams and Matlab/Simulink co-simulation environment. The results show that the patient 
unknown gait trajectory can be effectively tracked and the desired assistant training force can be properly produced. Therefore, the proposed Youla-Kucera parameterized adaptive regulator design approach can be used in LERR system to deal with the unknown factors and thus smoothly cooperate the patient's move intention during the rehabilitation training process.

\section{Acknowledgements}

This work was supported by the Hujiang Foundation of China(Grant No.B14002/D14002), the National Natural Science Foundation of China (Grant No.51275282) and Ph.D. Programs Foundation of Ministry of Education of China (Grant No.20123108110009).

\section{References}

[1] Y. Laufer, R. Dickstein, Y. Chefez and E. Marcovitz, "The Effect of Treadmill Training on the Ambulation of Stroke Survivors in the Early Stages of Rehabilitation:a randomized study", Journal of Rehabilitation Research and Development, vol. 38, no. 1, (2001), pp. 69-78.

[2] A. Duschau-Wicke, J. Von Zitzewitz, A. Caprez, L. Lünenburger and R. Riener, "Path control: A method for patient-cooperative robot-aided gait rehabilitation", IEEE Transactions on Neural system and Rehabilitation Engineering., vol. 18, no. 1, (2010), pp. 38-48.

[3] D. Delphine, L. Thierry and T. Jean-Louis, "The effect of repetitive rhythmic precision grip taskoriented rehabilitation in chronic stroke patients: a pilot study", International journal of rehabilitation Research, vol. 36, no. 1, (2013), pp. 81-87.

[4] L. M Wier, M. S. Hatcher, E. W. Triche and A. C. Lo, "Effect of robot-assisted versus conventional body-weight-supported treadmill training on quality of life for people with multiple sclerosis", Journal of Rehabilitation Research and Development, vol. 48, no. 4, (2011), pp. 483-492.

[5] H. Y. Sun, L. X. Zhang and C. S. Li, "Research on active compliance control and experimentation of a horizontal lower limbs rehabilitation robot", Chinese High Technology Letters., vol. 21, no. 6, (2011), pp. 624-628.

[6] R. Riener, L. Lünenburger, S. Jezernik, M. Anderschitz, G. Colombo and V. Dietz, "Patient-Cooperative Strategies for Robot-Aided Treadmill Training: First Experimental Results", IEEE Transactions on Neural Systems and Rehabilitation Engineering., vol. 13, no. 3, (2005), pp. 380-394.

[7] M. Bernhardt, M. Frey, G. Colombo and R. Riener, "Hybrid Force-Position Control Yields Cooperative Behaviour of the Rehabilitation Robot LOKOMAT", Proceedings of the IEEE 9th International Conference on Rehabilitation Robotics, (2005); Chicago, USA.

[8] M. S. Ju, C. C. K. Lin, D. H. Lin, I. S. Hwang and S. M. Chen, "A rehabilitation robot with forceposition hybrid fuzzy controller: hybrid fuzzy control of rehabilitation robot", IEEE Transactions on Neural Systems and Rehabilitation Engineering, vol. 13, no. 3, (2005), pp. 349-358.

[9] M. Pietrusinski, I. Cajigas, G. Severini, P. Bonato and C. Mavroidis, "Robotic Gait Rehabilitation Trainer", IEEE/ASME Transactions on Mechatronics, vol. 19, no. 2, (2014), pp. 490-499.

[10] L. Marchal-Crespo, "Converging Clinical and Engineering Research on Neurorehabilitation”, Edited J. L. Pons, D. Torricelli and M. Pajaro, Springer, Berlin Heidelberg, vol. 1, (2013), pp. 115-120.

[11] D. Erol and N. Sarkar, "Intelligent Control Framework for Robotic Rehabilitation after Stroke", IEEE International Conference on Robotics and Automation, (2007); Roma, Italy.

[12] A. Mayr, L. Luenenburger and E. Quirbach, "First experiences with the Lokomat in patients with severe traumatic brain injury”, Brain Injury, vol. 17, no. 1, (2003), pp. 46-47.

[13] Z. Feng, J. Qian, Y. Zhang and L. Shen, "Dynamics modeling and motion control for lower limb exoskeleton orthosis", Chinese High Technology Letters, vol. 19, no. 3, (2009), pp. 267-272.

[14] Z. Feng, “An Exoskeleton Robot for Gait Rehabilitation”, Shanghai University Publishers, Shanghai, (2009).

[15] S. Singh, "Decoupling of invertible nonlinear systems with state feedback and precompensation", IEEE Transactions on Automatic Control, vol. 25, no. 6, (1980), pp. 1237-1239.

[16] S. Singh, "A modified algorithm for invertibility in nonlinear systems", IEEE Transactions on Automatic Control, vol. 26, no. 2, (1981), pp. 595-598.

[17] Z. Zheng Wu and F. B. Amara, "Regulator synthesis for bimodal Linear Systems", IEEE Transactions on Automatic Control, vol. 56, no. 2, (2011), pp. 390-394.

[18] I. D. Landau, R. Lozano, M. M. Saad and A. Karimi, "Adaptive Control: Algorithm, Analysis and Applications", Springer-verlag, London, (2011). 


\section{Authors}
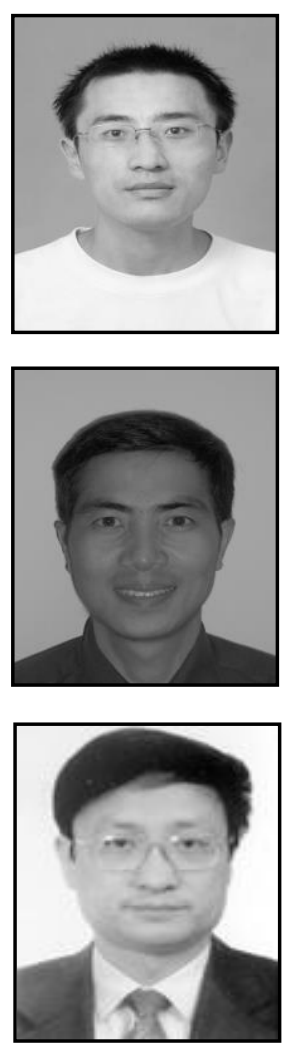

Feng $\mathbf{L i}$, is currently a lecturer in the school of optical electrical and computer Engineering, University of Shanghai for Science and Technology, China. His research interests include man-machine system and control of medical rehabilitation robot.

Zhizheng Wu, now is an associate professor in Shanghai University. His main research interests include adaptive control, optomechatronic systems and Robotics.

Jinwu Qian, is currently a professor and heads the Intelligent Machine and System Lab, Shanghai University, China. His main research interests include medical and rehabilitation robots, pipeline detecting robots, and gait analysis. 
International Journal of Control and Automation Vol.10, No.3 (2017) 